\title{
A preliminary modelling investigation into the safe correction zone for high tibial osteotomy
}

\author{
Jennifer L B Martay ${ }^{\mathrm{a}, *}$, Antony J R Palmer ${ }^{\mathrm{a}}$, Neil K Bangerter ${ }^{\mathrm{a}, \mathrm{b}}$, Stuart \\ Clare $^{\mathrm{c}}$, A Paul Monk ${ }^{\mathrm{a}}$, Cameron P Brown ${ }^{\mathrm{a}}$, Andrew J Price ${ }^{\mathrm{a}}$ \\ ${ }^{a}$ Nuffield Department of Orthopaedics, Rheumatology and Musculoskeletal Sciences, \\ University of Oxford, Oxford OX3 $7 L D, U K$ \\ ${ }^{b}$ Electrical $\& 3$ Computer Engineering, Brigham Young University, Provo, Utah 84602, \\ USA \\ ${ }^{c}$ Oxford Centre for Functional MRI of the Brain, Nuffield Department of Clinical \\ Neurosciences, University of Oxford, Oxford OX3 9DU, UK
}

\begin{abstract}
Purpose: High tibial osteotomy (HTO) re-aligns the weight-bearing axis (WBA) of the lower limb. The surgery reduces medial load (reducing pain and slowing progression of cartilage damage) while avoiding overloading the lateral compartment. The optimal correction has not been established. This study investigated how different WBA re-alignments affected load distribution in the knee, to consider the optimal post-surgery re-alignment. Methods: We collected motion analysis and 7T MRI data from 3 healthy subjects, and combined this data to create sets of subject-specific finite element models (total $=45$ models). Each set of models simulated a range of potential post-HTO knee re-alignments. We shifted the WBA from its native alignment to between $40 \%$ and $80 \%$ medial-lateral tibial width (corresponding to $2.8^{\circ}-3.1^{\circ}$ varus and $8.5^{\circ}-9.3^{\circ}$ valgus), in $3 \%$ increments. We then compared stress/pressure distributions in the models.

Results/Discussion: Correcting the WBA to $50 \%$ tibial width ( $0^{\circ}$ varusvalgus) approximately halved medial compartment stresses, with minimal changes to lateral stress levels, but provided little margin for error in undercorrection. Correcting the WBA to a more commonly-used 62\%-65\% tibial width $\left(3.4^{\circ}-4.6^{\circ}\right.$ valgus $)$ further reduced medial stresses but introduced the danger of damaging lateral compartment tissues. To balance optimal loading environment with that of the historical risk of under-correction, we propose a new target: WBA correction to $55 \%$ tibial width $\left(1.7^{\circ}-1.9^{\circ}\right.$ valgus $)$, which anatomically represented the apex of the lateral tibial spine.
\end{abstract}

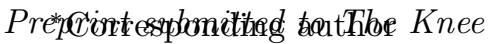

August 31, 2017

Email address: jennifer.boyd@oxon.org (Jennifer L B Martay) 
Conclusions: Finite element models can successfully simulate a variety of HTO re-alignments. Correcting the WBA to $55 \%$ tibial width $\left(1.7^{\circ}-1.9^{\circ}\right.$ valgus) optimally distributes medial and lateral stresses/pressures.

Keywords: Osteoarthritis, High tibial osteotomy, Weight-bearing axis, Finite element modelling, Knee alignment, Knee re-alignment

\section{Introduction}

Knee osteoarthritis (OA) is a chronic musculoskeletal disease of the tibiofemoral joint, and one of the leading causes of global disability [1]. Evidence suggests that the disease has a mechanical component. For example, approximately $75 \%$ of the compressive load in the knee passes through the medial compartment [2-3], and 90\% of cases of unicompartmental knee OA affect the medial tibiofemoral compartment [4]. Treatments which offload the medial compartment are therefore of great interest.

Opening wedge high tibial osteotomy (HTO) is an established, effective technique used to treat painful isolated medial compartment OA and limb mal-alignment [5-7]. HTO is a particularly attractive option for young individuals, allowing patients to resume high activity levels and delaying the need for arthroplasty [8-10]. The three-dimensional alteration of joint alignment during HTO transfers the position of the weight-bearing axis (WBA) from the affected medial compartment towards or into the normal lateral compartment of the knee (Fig 1). Biomechanically, this lateral shift decreases medial compartment stresses [11-12]. Historically, surgeons aimed to re-align the knee to between $3^{\circ}$ and $6^{\circ}$ valgus [5,13-18]. Re-alignment has also been described according to where the WBA crosses the tibial plateau: as a percentage of tibial width, measured from the medial side. Fugisawa et al recommend a target zone of $65 \%-70 \%$, which has been refined recently to $62.5 \%$ (range $62 \%-66 \%$ ) [19-20].

The outcome of HTO deteriorates with time, with around half remaining effective after seven years. The reasons for the unsuccessful outcomes are unclear but are thought to relate to inaccuracies in planning and surgical technique $[5,9,14,21]$. Despite the widespread use of the procedure, the optimal re-alignment of the WBA of the lower limb remains unknown [22]. A ten to thirteen-year follow-up study found 68 of 93 HTOs were undercorrected and led to continued medial compartment pain, while 5 of the 93 HTOs were overcorrected, resulting in the onset of lateral compartment pain 


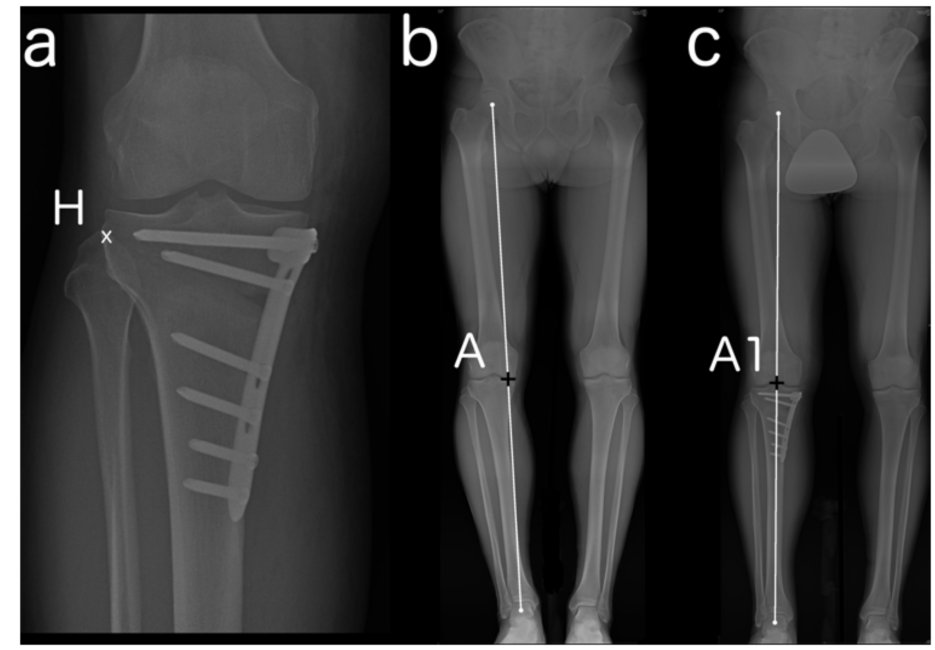

Figure 1: (a) Radiograph of a completed opening wedge HTO using a fixation plate. The osteotomy is made across the tibia and a wedge opened about the hinge point, H. (b, c) This moved the preoperative weight-bearing axis at the joint line more laterally (as demonstrated by the change from point 'A' to 'A1').

[23]. Under-correction of pre-operative deformity is an established predictor of failure, while moderate overcorrection appears to be desirable [23-24]. Recent reports suggest that improvements in instrumentation and navigated techniques will improve the accuracy of correction and inherent durability of HTO [25-28]. Understanding the relationship between re-alignment and the resulting stress redistributions will provide insights into future developments in surgical technique [29-30].

We describe the application of finite element (FE) analysis to investigate the relationship between a range of different WBA corrections and the associated stress and pressure distributions on the tibial plateau.

\section{Material and methods}

Ethical approval (09/H1102/88) for motion analysis was given by NRES Committee London. The MRI procedures were performed under an agreed technical development protocol (MSD/IDREC/2010/P17.2) approved by the Oxford University Clinical Trials and Research Governance office, in accordance with the International Electrotechnical Commission and United Kingdom Health Protection Agency guidelines. 


\subsection{Population information}

Motion analysis and MRI data were collected from 3 healthy subjects: 2 female, 1 male; aged 25-32 years; BMI 20.6-21.9; double support standing knee alignments $4.3^{\circ}-6.6^{\circ}$ varus. For this study, the following inclusion criteria were used: healthy subjects, 18 to 35 years (to decrease likelihood of musculoskeletal disease), with visually normal walking pattern and ability to sign informed consent. The following exclusion criteria were used: musculoskeletal pain within the previous year, previous musculoskeletal operation, contraindications for MRI scanning (metal in body, claustrophobic), inability to walk un-aided, and neuromuscular condition that could alter walking patterns.

Informed consent was given and absence of MRI contra-indications was verified prior to data collection. Please note that this preliminary study included a relatively small number of subjects who were not representative of typical HTO patients. The goal of this preliminary study was to investigate the feasibility of the specific modelling approach before potentially extending the procedure to a larger, more clinical patient cohort.

\subsection{Modelling technique}

For ease of reading, only an overview of methods is presented here; more details about the method are available in Appendix A. Motion analysis data was collected first. Retro-reflective markers were placed on each subject in an adapted Helen Hayes marker set [31], with seven additional registration markers placed around the knee to register the motion analysis and MRI data. Subjects then completed level walking cycles while marker trajectories and ground reaction forces (GRF) were recorded. MRI data was recorded next. Non-weight-bearing high resolution MRI scans (7 Tesla with a 28channel knee coil) were taken of the left knees in full extension. The seven additional registration markers were again present during MRI scanning.

The motion analysis and MRI data were then combined to create subjectspecific FE models of each individual subject during level walking. Bony and soft tissue geometries were obtained from manual segmentation of the MRI scans. Material properties were obtained from literature (Table A.1). Loading and alignment of the models were calculated from motion analysis data and GRF data at the point of maximum load in the walking cycle (Fig 2) because we assumed loading at this point in the walking cycle might lead to cartilage damage and lesions. After creating subject-specific models of native 
knee alignments, the models were modified to simulate a range of HTO realignments (Fig A.5). Simulations represented re-aligning the WBA to pass between $40 \%$ and $80 \%$ medio-lateral (M-L) tibial width, in $3 \%$ increments. This range corresponded to knee alignments of between $2.8^{\circ}-3.1^{\circ}$ varus and $8.5^{\circ}-9.3^{\circ}$ valgus in our subjects. In total, 45 models were created $(15 \mathrm{FE}$ models per subject).

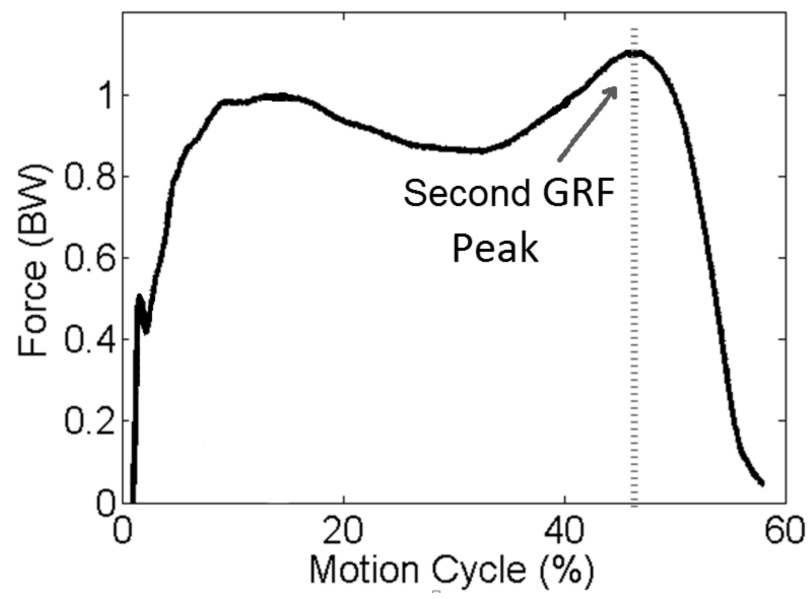

Figure 2: Maximum loads during level walking, a common activity, are experienced during the second peak of the ground reaction force (GRF) data. The FE models represent loading at this time point.

We chose to use FE models because the models gave the opportunity to non-invasively pre-plan an HTO surgery, and consider the effects of realigning the knee to a range of different positions. Making the models subject-specific let us investigate the optimal correction zone for an individual subject. This was important to us in case different subjects might have different optimal correction zones.

\subsection{Outcomes measures}

Von Mises stresses and contact pressures on the articulating tibial cartilage and meniscal surfaces were extracted from each model and compared (more details in Appendix B).

\subsection{Statistical analysis}

This study did not include statistical tests due to low subject numbers $(\mathrm{n}=3)$. When pooling results, the average and standard error of the mean 
were calculated. The standard error was used as an estimate for the standard deviation, again due to low subject numbers. Instead of using statistics, we considered the relationship between stresses and pressures in the medial and lateral compartments when determining an optimal correction zone.

\subsection{Porcine validation study}

Due to limitations on using human cadavers in our laboratory, we initially validated our model creation method using porcine specimens, before comparing our results to a published human cadaveric study. A detailed explanation of the porcine validation study is available in reference [32]. As an overview, pressure film was inserted into two porcine knees while known loads were applied to the knees. The pressures experienced within the knees were recorded. The knees were then fixed in their positions and imaged using an MRI scanner. Knee geometry from the MRI scans and load conditions from the experimental tests were then combined to create subject-specific FE models, and the FE models were solved for contact pressures. There was good agreement between the pressures measured experimentally by the pressure film and the pressures calculated by the FE models.

\section{Results}

The three subjects had native alignments with the WBA passing through $27 \%, 27 \%$, and $21 \%$ M-L tibial width $\left(7.1^{\circ}, 6.5^{\circ}\right.$, and $8.9^{\circ}$ varus $)$ at the point of maximum GRF. The loads calculated for the range of different realignments were: Subject 1) $756.8 \mathrm{~N}$ with $30.1 \mathrm{Nm}$ varus to $13.5 \mathrm{Nm}$ valgus moments; Subject 2) $766.0 \mathrm{~N}$ with $31.9 \mathrm{Nm}$ varus to $10.0 \mathrm{Nm}$ valgus moments; and Subject 3) $667.8 \mathrm{~N}$ with $35.1 \mathrm{Nm}$ varus to $8.2 \mathrm{Nm}$ valgus moments.

Areas of high stress and pressure moved from the medial to the lateral compartment as the WBA was shifted laterally. Representative pressure distributions for different re-alignments in a single subject are shown in Fig 3. In the native alignment, high pressures were found in the medial compartment, particularly in the anterior regions and on the meniscus. As the WBA shifted incrementally laterally, the femoral cartilage reduced contact with the medial tibial cartilage, increased contact with the lateral meniscus, and then increased contact with the lateral tibial cartilage. Similar transitions of both contact areas and stress and pressure magnitudes were observed in each subject. 

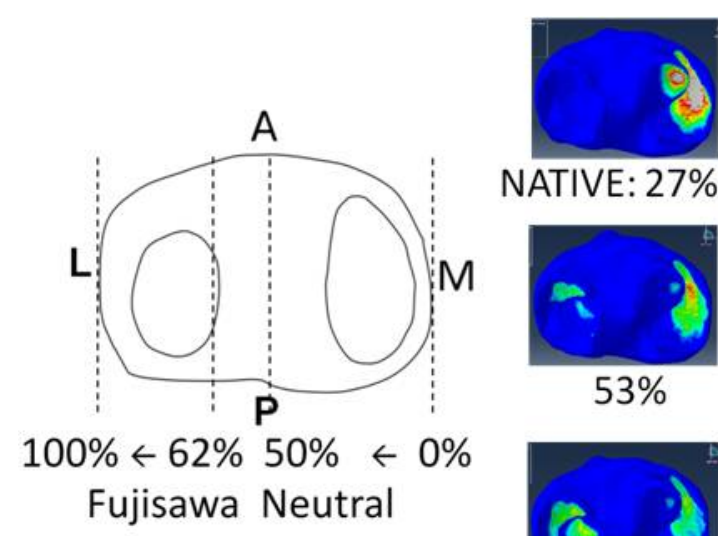

NATIVE: $27 \%$

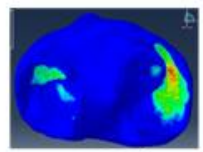

$53 \%$

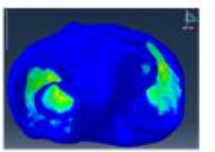

$68 \%$

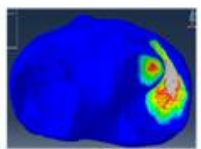

$40 \%$

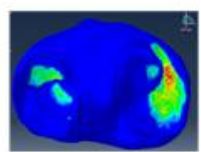

$56 \%$

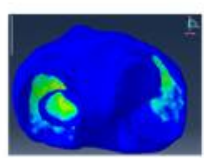

$71 \%$

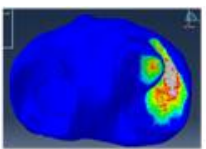

$44 \%$

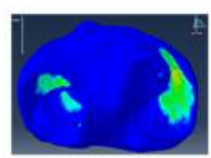

$59 \%$

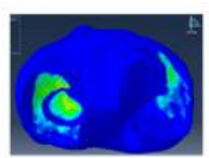

$74 \%$

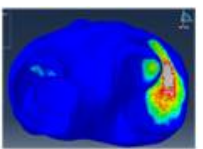

$47 \%$

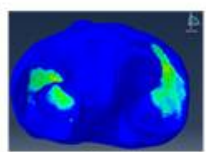

$62 \%$

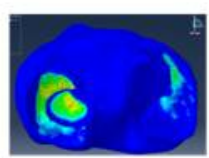

$77 \%$

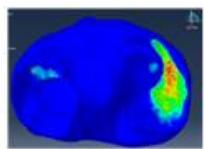

$50 \%$

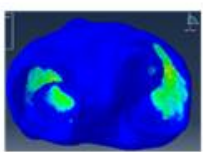

$65 \%$

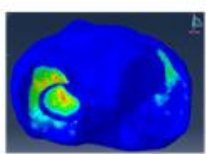

$80 \%$

Figure 3: (left) Models simulated incrementally shifting the WBA from the medial to lateral compartment; (right) Example of contact pressures on articulating tibial cartilage and meniscal surfaces. High pressures moved from medial to lateral compartment as WBA shifted laterally (all figures use same color threshold [0-4 MPa]; higher pressures in $\mathrm{red} / \mathrm{grey})$.

Trends were also similar when all subjects were pooled (Fig 4). Shifting the WBA laterally decreased medial compartment pressures/stresses and increased lateral compartment pressures/stresses, on both the cartilage and meniscal surfaces (Figs B.6 and B.7). Medial compartment pressures remained relatively large if only small corrections were simulated (the WBA was re-aligned to less than $50 \% \mathrm{M}$-L tibial width and remained in varus alignment). In a neutral ( $50 \% \mathrm{M}-\mathrm{L}$ tibial width, $0^{\circ}$ varus-valgus) alignment, medial pressures decreased to approximately $55 \%$ of their maximum values, with negligible increases in lateral compartment pressures. Re-aligning to the Fujisawa point ( $62 \%-65 \% \mathrm{M}-\mathrm{L}$ tibial width, $3.4^{\circ}-4.6^{\circ}$ valgus) further decreased medial pressures to approximately $40 \%$ of their maximum values, with noticeably increased lateral pressures. The intersection of decreasing medial and increasing lateral pressures occurred at approximately $60 \%$ realignment $\left(2.6^{\circ}-2.8^{\circ}\right.$ valgus $)$.

\section{Discussion}

The aim of this study was to propose a safe correction zone for HTO re-alignments. By comparing medial and lateral compartment 

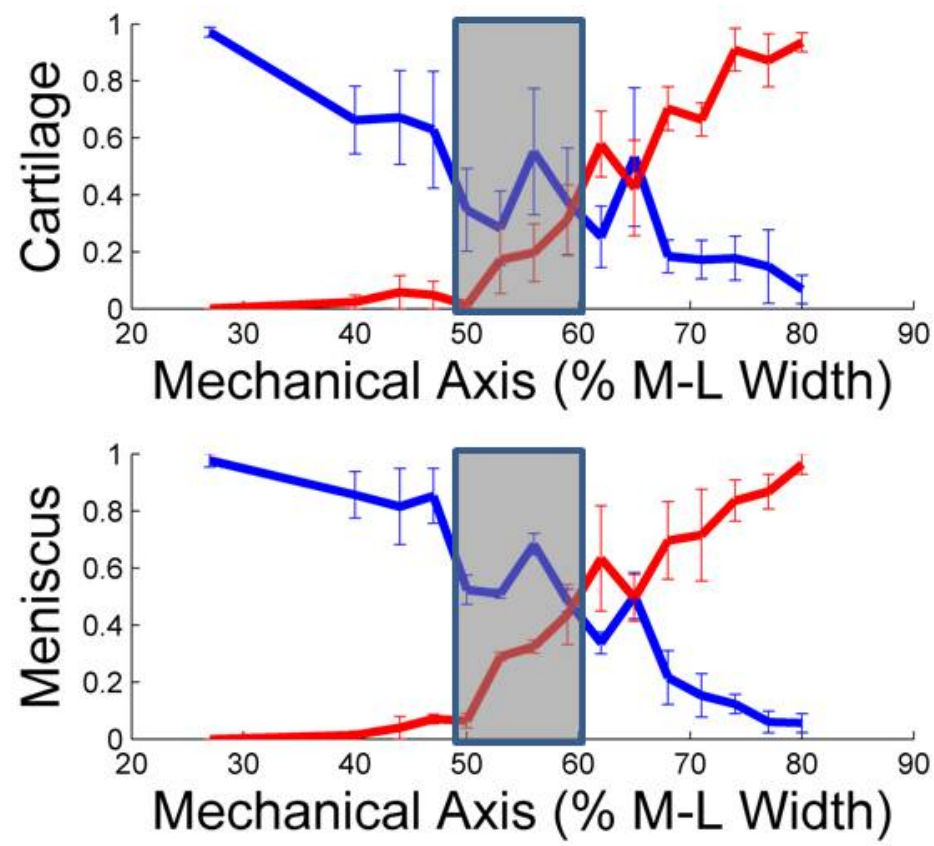

Figure 4: Averages and standard errors of normalized pressures on the (blue) medial and (red) lateral (top) cartilage and (bottom) meniscal surfaces. Proposed 'safe' zone shaded in grey. $\mathrm{M}-\mathrm{L}=$ medial-lateral.

stresses/pressures, we found a repeatable safe zone for our three subjects: aiming for the WBA to cross at $55 \%$ tibial width $\left(1.7^{\circ}-1.9^{\circ}\right.$ valgus $)$.

In the native alignment of the knee, the WBA crossed in the medial compartment (21\% to $27 \% \mathrm{M}-\mathrm{L}$ tibial width, $6.5^{\circ}-8.9^{\circ}$ varus), resulting in stress/pressure only being experienced in the medial compartment. Our results agree with the assumptions and results of other studies [33-36].

Re-aligning the WBA to cross the knee at less than 50\% M-L tibial width $\left(0^{\circ}\right.$ varus-valgus $)$ had little effect on medial pressures. This range of re-alignments could be classified as 'undercorrections'. Contact area and stress/pressure magnitudes remained similar to their pre-HTO states. Therefore, re-aligning the WBA to less than 50\% M-L tibial width (remaining in varus) will have limited benefit for the patient (in agreement with reference [23]).

At the other extreme, re-aligning the WBA to cross beyond $65 \% \mathrm{M}-\mathrm{L}$ tibial width $\left(4.3^{\circ}-4.6^{\circ}\right.$ valgus $)$ resulted in large lateral stresses and pressures. This range of re-alignments could be classified as 'overcorrections'. In this 
range of re-alignments, lateral tissues are now susceptible to damage, and patients may develop lateral compartment pain (as described in reference [23]).

Re-aligning the WBA to cross between $50 \%$ and $60 \%$ M-L tibial width $\left(0^{\circ}\right.$ varus-valgus to $2.6^{\circ}-2.8^{\circ}$ valgus) could therefore be considered a middle ground, or a 'safe zone'. Within this zone, medial compartment stress and pressure decreased without substantial increases in the lateral compartment. We selected an upper range of $60 \%$, rather than $65 \%$, because of the potential for small errors during surgery. For example, if refined Fugisawa re-alignments of $62.5 \%$ were attempted, small errors in over-correction during surgery might result in actual post-HTO re-alignments beyond $65 \%$, which could increase lateral compartment pressures to unacceptable, damaging levels [19-20]. An alternative and potentially safer re-alignment would be to $55 \%$ M-L tibial width $\left(1.7^{\circ}-1.9^{\circ}\right.$ valgus $)$. With this re-alignment, the surgeon can have a margin of error of $5 \%$ above and below and still be within the safe zone. The $55 \%$ point corresponds to the apex of the lateral spine, producing an easily identifiable anatomical target.

\subsection{Assumptions and Limitations}

This study calculated stress and pressure distributions for a variety of simulated HTO re-alignments. This raises a question, however, about how large the stress/pressure magnitudes need to be to cause damage initiation and/or progression. The extent to which medial compartment pressures and stresses must be reduced to prevent progression is unknown, as is the threshold beyond which increasing lateral compartment pressures and stresses will initiate damage. In this study, we assumed that if a patient requires an HTO, their medial compartment stresses/pressures are already 'too high', and so we have sought to lower those medial stresses/pressures. Simultaneously, we sought to maintain relatively low lateral stresses/pressures to avoid damaging the lateral tissues.

Another limitation is that our models used simple material behaviors to examine macroscale patterns of stress and pressure. This study aimed to test the feasibility of our method and to explore the effects different knee realignments had on stress/pressure. Therefore, simple material behaviors were deemed acceptable for our current study. The increased structural specificity needed to explore damage in cartilage, menisci, and bone is outside the scope of this study, yet warrants further examination. 
Validation is an important aspect of any study involving FE models. We initially validated our model creation method using porcine specimens (details provided in Section 2.5), before comparing our results to a published human cadaveric study which focused on HTO and knee re-alignments [37]. Both our current study and the cadaveric study used FE models to simulate the effects of knee re-alignment on tibiofemoral pressure distribution. Model set-ups were similar, and both studies simulated knnee re-alignments by varying the knee adduction-abduction moment. A major difference between our current study and the cadaveric study was that the cadaveric study's models included 3D pre-tuned ligaments, whereas our study included axial ligaments with literature-derived material properties. Our current study further differs from the cadaveric study in that we used subject-specific loads measured during walking. The human cadaveric study applied loads of $374 \mathrm{~N}$ with $15 \mathrm{Nm}$ varus to $15 \mathrm{Nm}$ valgus moments. Therefore, our applied loads and maximum varus moments were approximately double those used in the human cadaveric study, while our maximum valgus moments ranged from nearly the same to half. Despite these differences in the two studies, trends in medial-lateral pressure distributions were similar. Through this indirect comparison, we conclude that our results appear reasonable and have been at least partially validated.

\section{Conclusions}

The philosophy behind HTO is to re-align the WBA to offload medial stresses (with the aim of reducing pain and slowing progression of cartilage damage) while avoiding overloading the lateral compartment. This study revealed that re-aligning the WBA to $50 \%$ M-L tibial width ( $0^{\circ}$ varus-valgus) reduced medial compartment stresses by approximately half, with very small changes to lateral stress levels, yet provided little margin for error in undercorrection. Durability of HTO beyond seven years has been linked historically to moderate over-correction $[22,24]$, corresponding with our finding that a 50\% re-alignment might not be sufficient. Furthermore, it appears that correcting the WBA to a more commonly-used $62 \%-65 \%$ M-L tibial width $\left(3.4^{\circ}-4.6^{\circ}\right.$ valgus $)$ further reduced medial stresses but introduced the potential to damage the tissues in the lateral compartment.

To balance optimal loading environment with that of the risk of undercorrection, we propose a new target WBA correction to $55 \%$ M-L tibial width 
$\left(1.7^{\circ}-1.9^{\circ}\right.$ valgus). This correction anatomically represents a point at the apex of the lateral tibial spine.

\section{References}

[1] Cross M, Smith E, Hoy D, Nolte S, Ackerman I, Fransen M, et al. The global burden of hip and knee osteoarthritis: Estimates from the Global Burden of Disease 2010 study. Annals of the rheumatic diseases 2014;73(7):1323-30.

[2] Andriacchi TP. Dynamics of knee malalignment. The orthopaedic clinics of North America 1994;25(3):395-403.

[3] Perie D, Hobatho MC. In vivo determination of contact areas and pressure of the femorotibial joint using non-linear finite element analysis. Clinical biomechanics 1998;13(6):394-402.

[4] Hernborg JS, Nilsson BE. The natural course of untreated osteoarthritis of the knee. Orthopaedics 1977;123:130-7.

[5] Flecher X, Parratte S, Aubaniac JM, Argenson JN. A 12-28-year followup study of closing wedge high tibial osteotomy. Clinical orthopaedics and related research 2006;452:91-6.

[6] Jackson JP, Waugh W, Green JP. High tibial osteotomy for osteoarthritis of the knee. The journal of bone and joint surgery British volume 1969;51(1):88-94.

[7] Seil R, van Heerwaarden R, Lobenhoffer P, Kohn D. The rapid evolution of knee osteotomies. Knee surgery, sports traumatology, arthroscopy: official journal of the ESSKA 2013;21(1):1-2.

[8] Diduch DR, Insall JN, Scott WN, Font-Rodriguez D. Total knee replacement in young, active patients. Long-term follow-up and functional outcome. The journal of bone and joint surgery American volume 1997;79(4):575-82. [9] Insall JN, Joseph DM, Msika C. High tibial osteotomy for varus gonarthrosis. A long-term follow-up study. The journal of bone and joint surgery American volume 1984;66(7):1040-8.

[10] Nagel A, Insall JN, Scuderi GR. Proximal tibial osteotomy. A subjective outcome study. The journal of bone and joint surgery American volume 1996;78(9):1353-8.

[11] Johnson F, Leitl S, Waugh W. The distribution of load across the knee. A comparison of static and dynamic measurements. The journal of bone and joint surgery British volume 1980;62(3):346-9.

[12] Maquet P. The biomechanics of the knee and surgical possibilities of 
healing osteoarthritic knee joints. Clinical orthopaedics and related research 1980;146:102-10.

[13] Akizuki S, Shibakawa A, Takizawa T, Yamazaki I, Horiuchi H. The long-term outcome of high tibial osteotomy: A ten- to 20-year follow-up. The journal of bone and joint surgery British volume 2008;90(5):592-6. [14] Coventry MB. Osteotomy of the upper portion of the tibia for degenerative arthritis of the knee. A preliminary report. The journal of bone and joint surgery American volume 1965;47:984-90.

[15] Kettelkamp DB, Wenger DR, Chao EY, Thompson C. Results of proximal tibial osteotomy. The effects of tibiofemoral angle, stance-phase flexion-extension, and medial-plateau force. The journal of bone and joint surgery American volume 1976;58(7):952-60.

[16] Koshino T, Yoshida T, Ara Y, Saito I, Saito T. Fifteen to twenty-eight years' follow-up results of high tibial valgus osteotomy for osteoarthritic knee. The knee 2004;11(6):439-44.

[17] Papachristou G, Plessas S, Sourlas J, Levidiotis C, Chronopoulos E, Papachristou C. Deterioration of long-term results following high tibial osteotomy in patients under 60 years of age. International orthopaedics 2006;30(5):403-8.

[18] Tang WC, Henderson IJ. High tibial osteotomy: Long term survival analysis and patients' perspective. The knee 2005;12(6):410-3.

[19] Dugdale TW, Noyes FR, Styer D. Preoperative planning for high tibial osteotomy. The effect of lateral tibiofemoral separation and tibiofemoral length. Clinical orthopaedics and related research 1992;274:248-64.

[20] Fujisawa Y, Masuhara K, Shiomi S. The effect of high tibial osteotomy on osteoarthritis of the knee. An arthroscopic study of 54 knee joints. The orthopedic clinics of North America 1979;10(3):585-608.

[21] DeMeo PJ, Johnson EM, Chiang PP, Flamm AM, Miller MC. Midterm follow-up of opening-wedge high tibial osteotomy. The American journal of sports medicine 2010;38(10):2077-84.

[22] Wright JM, Crockett HC, Heber C, Slawski DP, Madsen MW, Windsor RE. High tibial osteotomy. The journal of the American Academy of Orthopaedic Surgeons 2005;13(4):279-89.

[23] Hernigou P, Medevielle D, Debeyre J, Goutallier D. Proximal tibial osteotomy for osteoarthritis with varus deformity: A ten to thirteen-year follow-up study. The journal of bone and joint surgery American version 1987;69(3):332-54.

[24] Majima T, Yasuda K, Katsuragi R, Kaneda K. Progression of joint 
arthrosis 10 to 15 years after high tibial osteotomy. Clinical orthopaedics and related research 2000;381:177-84.

[25] Gaasbeek RD, Nicolaas L, Rijnberg WJ, van Loon CJ, van Kampen A. Correction accuracy and collateral laxity in open versus closed wedge high tibial osteotomy. A one-year randomised controlled study. International orthopaedics 2010;34(2):201-7.

[26] Hankemeier S, Hufner T, Wang G, Kendoff D, Zeichen J, Zheng G, et al. Navigated open-wedge high tibial osteotomy: Advantages and disadvantages compared to the conventional technique in a cadaver study. Knee surgery, sports traumatology, arthroscopy: official journal of the ESSKA 2006;14(10):917-21.

[27] Hankemeier S, Mommsen P, Krettek C, Jagodzinski M, Brand J, Meyer $\mathrm{C}$, et al. Accuracy of high tibial osteotomy: Comparison between open- and closed-wedge technique. Knee surgery, sports traumatology, arthroscopy: official journal of the ESSKA 2010;18(10):1328-33.

[28] Keppler P, Gebhard F, Grutzner PA, Wang G, Zheng G, Hüfner T, et al. Computer aided high tibial open wedge osteotomy. Injury 2004; 35 Suppl 1:S-A68-78.

[29] Billings A, Scott DF, Camargo MP, Hofmann AA. High tibial osteotomy with a calibrated osteotomy guide, rigid internal fixation, and early motion. Long-term follow-up. The journal of bone and joint surgery American volume 2000;82(1):70-9.

[30] Sprenger TR, Doerzbacher JF. Tibial osteotomy for the treatment of varus gonarthrosis. Survival and failure analysis to twenty-two years. The journal of bone and joint surgery American volume 2003;85(3):469-74.

[31] Kadaba MP, Ramakrishnan HK, Wootten ME. Measurement of lower extremity kinematics during level walking. Journal of orthopaedic research 1990;8(3):383-92.

[36] Boyd JL, Zavatsky AB, Gill HS. Does increasing applied load lead to contact changes indicative of knee osteoarthritis? A subject-specific FEA study. International journal of numerical methods in biomedical engineering 2016;32(4).

[33] Harrington IJ. A bioengineering analysis of force actions at the knee in normal and pathological gait. Biomedical Engineering 1976;11(5):167-72.

[34] Morrison JB. The mechanics of the knee joint in relation to normal walking. Journal of biomechanics 1970;3(1):51-61.

[35] Schipplein OD, Andriacchi TP. Interaction between active and passive knee stabilizers during level walking. Journal of orthopaedic research 
1991;9(1):113-9.

[36] Shelburne KB, Torry MR, Pandy MG. Contributions of muscles, ligaments, and the ground-reaction force to tibiofemoral joint loading during normal gait. Journal of orthopaedic research 2006;24(10):1983-90.

[37] Mootanah R, Imhauser CW, Reisse F, Carpanen D, Walker RW, Koff $\mathrm{MF}$, et al. Development and validation of a computational model of the knee joint for the evaluation of surgical treatments for osteoarthritis. Computer methods in biomechanics and biomedical engineering 2014;17(13):1502-17. [38] Wengert C, Bianchi G. Absolute orientation: http://www.mathworks.com/matlabcentral/fileexchange/22422absoluteorientation Matlab File Exchange accessed 01 August 2011. [39] Haut Donahue TL, Hull ML, Rashid MM, Jacobs CR. A finite element model of the human knee joint for the study of tibio-femoral contact. Journal of biomechanical engineering 2002;124(3):273-80.

[40] Bae J, Park K, Seon J, Kwak DS, Jeon I, Song EK. Biomechanical analysis of the effects of medial meniscectomy on degenerative osteoarthritis. Medical \& biological engineering \& computing 2012;50(1):53-60. [41] Yang NH, Canavan PK, Nayeb-Hashemi H, Najafi B, Vaziri A. Protocol for constructing subject-specific biomechanical models of knee joint. Computer methods in biomechanics and biomedical engineering 2010;13(5):589-603.

[42] Atkinson P, Atkinson T, Huang C, Doane R. A comparison of the mechanical and dimensional properties of the human medial and lateral patellofemoral ligaments. Proceedings of the 46th Orthopedic Research Society. 2000.

[43] Butler DL, Kay MD, Stouffer DC. Comparison of material properties in fascicle-bone units from human patellar tendon and knee ligaments. Journal of biomechanics 1986;19(6):425-32.

[44] Trent PS, Walker PS, Wolf BW. Ligament length patterns, strength, and rotational axes of the knee joint. Clinical orthopaedics and related research 1976;117:263-270.

\section{Acknowledgements}

Dr Julie Stebbins is thanked for her assistance in marker placement. Dr Peter Jezzard is thanked for his assistance with MRI data acquisition. 


\section{Funding}

This study was funded by Arthritis Research UK (ref 20299 and Oxford EOTC), Orthopaedic Research UK (ref 504), and the Oxford NIHR Biomedical Research Unit.

\section{Appendix A. Detailed Methods}

\section{Appendix A.1. Data Collection and Processing}

Motion analysis began by recording each subject's height, weight, lower limb lengths, distances between anterior-superior iliac spine prominences, and widths of the ankles and knees. Thirty-three retro-reflective markers were placed on bony landmarks according to an adapted Helen Hayes marker set [31]. Seven additional markers were placed around the knee, and were later used to register the motion analysis and MRI data. Subjects then completed five cycles, each, of level walking (self-selected speed) within the capture volume of a 12-camera video-based motion analysis system. Marker trajectory data (Vicon 612, Vicon Motion Systems Ltd., Oxford, UK) and GRF data (OR6 platform, Advance Mechanical Technology Inc., Watertown, MA, USA) were collected during these walking cycles.

Immediately following motion analysis, MRI scans were collected. The seven additional registration markers were replaced by fluid-filled MRI registration markers (MM3002 Multi-Modality Fiducial Markers, IZI Medical, Owings Mills, MD, USA). Separate non-weight-bearing MRI scans (custom 3D phase-cycled balanced steady-state free precession pulse sequence, $\mathrm{TR} / \mathrm{TE} 5.73 \mathrm{~ms} / 2.47 \mathrm{~ms}, \mathrm{FA}=20^{\circ}, \mathrm{FOV}=160 \mathrm{~mm} \times 160 \mathrm{~mm} \times 11.3$ $\mathrm{mm}$, resolution: $0.31 \mathrm{~mm} \times 0.31 \mathrm{~mm} \times 0.31 \mathrm{~mm}, 2$ phase cycles with sumof-squares combination, Siemens 7 Tesla field strength whole body scanner, Siemens Healthcare, Surry, UK) were taken of the left knees in full extension. MRI data was segmented (ITK-SNAP 2.4.0, Cognitica Corporation and National Library of Medicine, Bethesda, MD, USA) according to voxel greyscale intensity values to obtain the 3D surfaces of the tibia, femur, patella, tibial cartilage, femoral cartilage, patellar cartilage, and menisci. The seven registration marker locations were also identified. The segmented structure surfaces were checked for integrity and discontinuities (Blender 2.68a, Stichting Blender Foundation, Amsterdam, The Netherlands), and the resulting 
structures were assembled to create the knee model geometry (SolidWorks 2014, Dassault Systemes SolidWorks Corp., Concord, MA, USA).

The MRI data and motion analysis data were originally collected in different coordinate systems. The two data sets were matched/registered (leastsquares error minimization [38]) using the seven registration markers included during both types of data collection. The calculated loads (derived from the motion analysis data) were then transformed into the MRI coordinate system (Matlab R2014a, MathWorks, Natick, MA, USA).

\section{Appendix A.2. FE Model Creation}

Subject-specific geometries and loads were combined to create the FE models (Abaqus 6.12, Dassault Systemes, Providence, RI, USA). First, subject-specific FE models of the subjects in native knee alignments were created. Models included bones, cartilage, menisci, ligaments, posterior capsule, and quadriceps muscles.

The material properties used in the FE models were derived from the literature (Table A.1). Bone is much stiffer than cartilage and menisci. Therefore, we modeled bone as a rigid material [39] to speed up model solution. Cartilage and menisci are both viscoelastic materials. The elapsed loading times during walking are significantly shorter than these tissues' viscoelastic time constants ( 1500 seconds [39]), however, so fluid would not have moved within these tissues during these short elapsed loading times. Therefore, we modeled cartilage and menisci as linear elastic isotropic and linear elastic transversely isotropic materials, respectively [40-41]. Ligaments were modelled as uniaxial connectors (force-extension properties from references [42-44]), with connector ends placed at the origin and insertion sites identified in the MRI scans.

Loads were derived from GRF data, collected at the foot-ground interface. In equilibrium and in single stance (only a single foot contacting the ground, as occurs at the second GRF peak), the loads experienced at the foot-ground interface will be equal and opposite the loads experienced at the femur. Our 7T MRI scans could not image the entire lower limb at once, however, so the foot-ground interface was not included in our FE models. Instead, we applied an equivalent force system to the femur, specifically to the femoral centre of mass.

The models' boundary and contact conditions reflected the conditions in the physiological knee. The tibia was held fixed (while loads were applied 


\begin{tabular}{|l|c|c|}
\hline STRUCTURE & PARAMETERS & REF \\
\hline Bone & Rigid & {$[39]$} \\
\hline Cartilage & $\mathrm{E}=15 \mathrm{MPa}, \mathrm{v}=0.45$ & {$[41]$} \\
\hline Menisci: Circumferential & $\mathrm{E}=120 \mathrm{MPa}, \mathrm{v}=0.3$ & {$[41]$} \\
Axial & $\mathrm{E}=20 \mathrm{MPa}, \mathrm{v}=0.2$ & {$[41]$} \\
\hline Ligaments: ACL, PCL, MCL, LCL & Force-displacement curves & {$[44]$} \\
Patellofemoral & Force-displacement curves & {$[42]$} \\
Patellar tendon & Force-displacement curves & {$[43]$} \\
\hline Quadriceps muscle & Force-displacement curve for patellar tendon & {$[43]$} \\
\hline Posterior capsule & Force-displacement curve for LCL & {$[44]$} \\
\hline
\end{tabular}

Table A.1: FE model material properties

to the femur). As in the physiological knee, the meniscal horns were tied to the tibia. Bone-cartilage interactions were tied to represent the physiological calcified cartilage region. Soft tissue interactions (cartilage-cartilage and cartilage-menisci) were modeled as frictionless and allowed articulating surfaces to separate after coming into contact.

After creating the subject-specific models of the native WBA alignments, additional models were created to represent re-aligning the WBA to different positions. The same geometry was used in all models. The applied loads were changed to reflect different re-alignments. HTO re-aligns the knee and shifts the WBA from the medial compartment towards the tibial spine/lateral compartment. In equivalent force systems, shifting a force $(\mathrm{F})$ to a location off its line of action $(\Delta \mathrm{d})$ creates a moment $(\mathrm{M}=\Delta \mathrm{d} \times \mathrm{F})$ (Fig A.5). Therefore, different WBA re-alignments were simulated by applying different adduction-abduction moments to the models (applied loads were held constant since HTO does not significantly affect body weight). These additional FE models were created with moments representing moving the WBA from $40 \%$ to $80 \%$ M-L tibial width, in $3 \%$ increments. The equivalent varus-valgus angle of each knee was also calculated.

\section{Appendix B. Analysis of Model Outputs}

Von Mises stresses and contact pressures on the articulating tibial cartilage and meniscal surfaces were extracted from each model. First, the effects of re-aligning the WBA to different locations was considered for each individual subject. Then the average patterns for the three subjects were considered. 

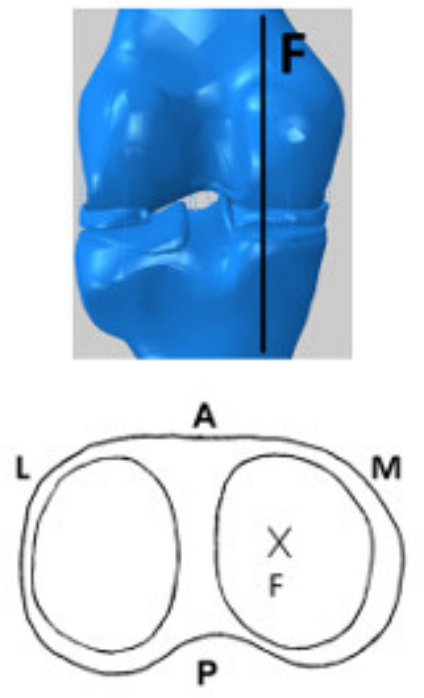

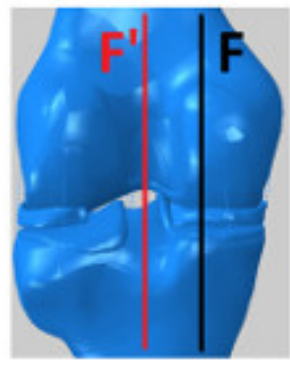

A

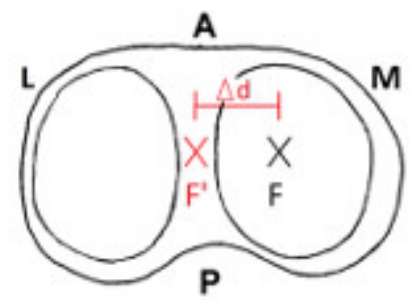

Figure A.5: Models were first created with the knee in its (left) native alignment (WBA force F shown in black). (right) Simulated HTO models were then created by shifting the WBA from the native alignment (black, F) towards the lateral compartment (red, F'). Shifting the WBA creates an adduction-abduction moment.

When comparing models for a single subject, the distribution patterns of stress and pressure were first visually inspected. Locations of highest stress and pressure were noted. Trends of how stresses and pressures shifted between the medial and lateral compartment as WBA shifted laterally were also noted.

Magnitudes of stresses and pressures were then quantitatively compared. Stress and pressure magnitudes were recorded in four regions of interest: cartilage-cartilage and cartilage-meniscus contact areas, in both the medial and lateral compartment. Regions were defined in the models with the largest contact area: native alignment models for medial regions and $80 \%$ mediallateral tibial width models for lateral regions. The average stress and average pressure within each of these four regions was calculated for each model, and then plotted against knee re-alignment to investigate how medial and lateral results were affected by knee re-alignment.

Next, individual subject results were pooled to investigate overall stress/pressure re-alignment trends. Each subject had different maximum stress and pressure values, so the stresses and pressures were first normalized according to each subject's maximum results. Maximum medial 
stresses/pressures occurred in the native alignment models; maximum lateral stresses/pressures occurred in the $80 \%$ medial-lateral re-alignment models. After normalizing to each subject's maximum value, the normalized results of the three subjects were averaged. Line graphs (Figs 4 and B.6) and bar plots (Fig B.7) were created to visualize the normalized and relative stresses and pressures in the four regions of interest, and to investigate how stress/pressure shifted between the medial and lateral compartments as the WBA shifted laterally.

\section{Appendix B.1. Additional Results}

Stresses and pressures on the cartilage and menisci surfaces, as well as relative stresses and pressures on the articulating surfaces are shown in Figures B.6 and B.7.
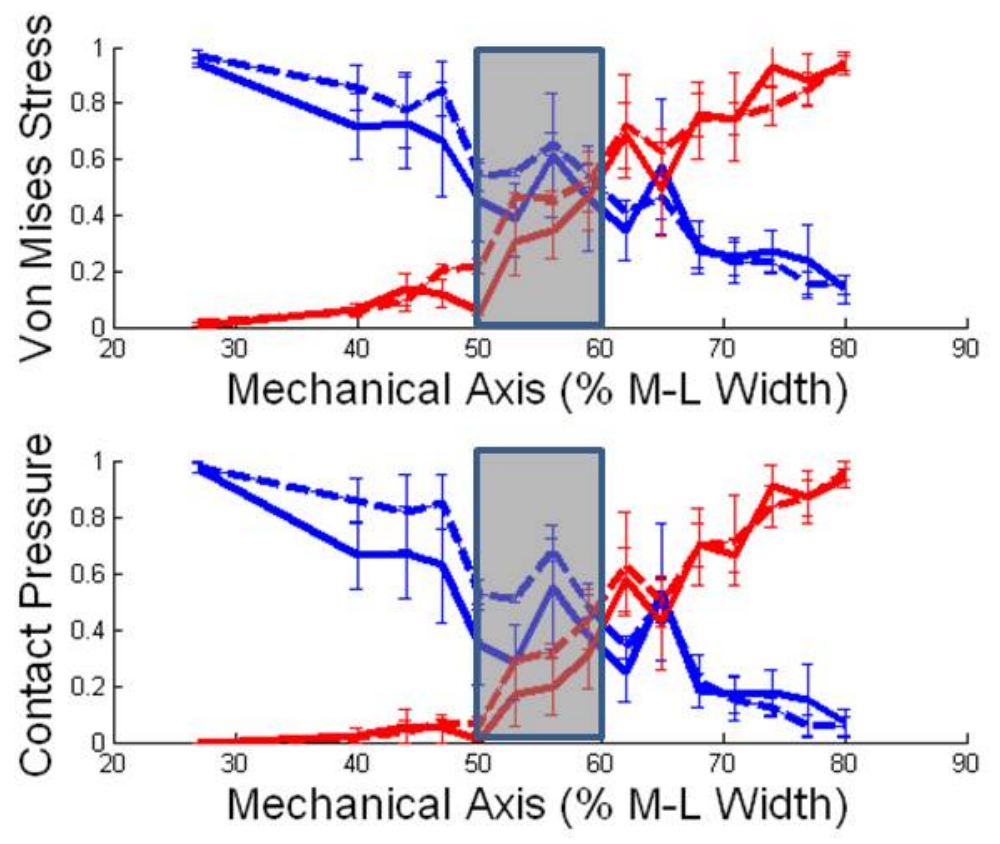

Figure B.6: Averages and standard errors of normalized von Mises stress and contact pressure on the articulating (solid lines) cartilage and (dashed lines) meniscal surfaces of the (blue) medial and (red) lateral compartments. Proposed 'safe' zone shaded in grey. $\mathrm{M}-\mathrm{L}=$ medial-lateral. 

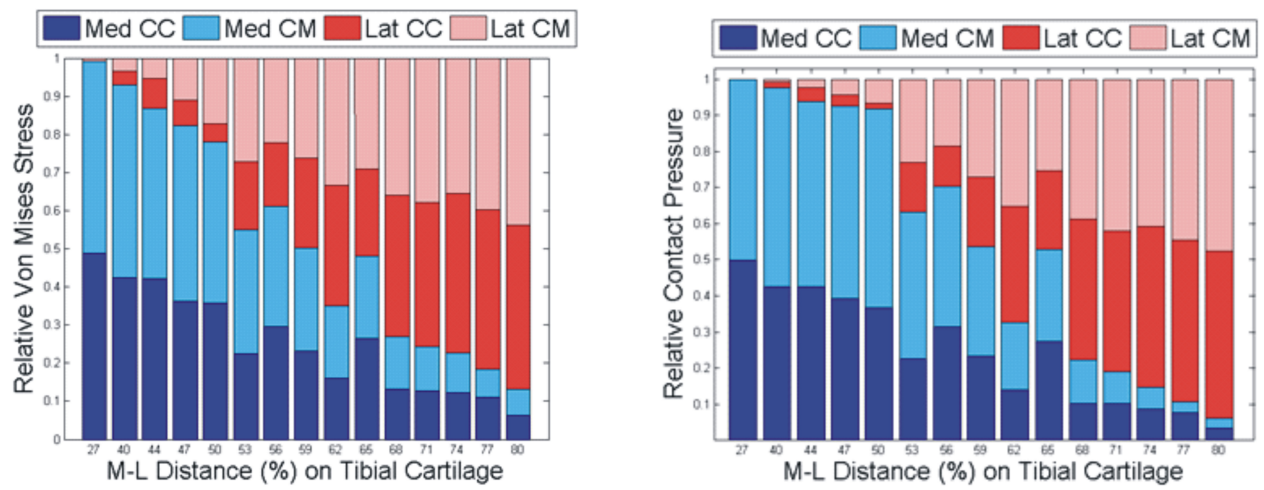

Figure B.7: Relative (top) stresses and (bottom) pressures on the (blue) medial and (red) lateral tibial cartilage and menisci. $\mathrm{M}-\mathrm{L}=$ medial-lateral.

\section{Appendix C. Figure legends}

Figure 1: (a) Radiograph of a completed opening wedge HTO using a fixation plate. The osteotomy is made across the tibia and a wedge opened about the hinge point, $\mathrm{H}$. (b, c) This moved the preoperative weight-bearing axis at the joint line more laterally (as demonstrated by the change from point 'A' to 'A1').

Figure 2: Maximum loads during level walking, a common activity, are experienced during the second peak of the ground reaction force (GRF) data. The FE models represent loading at this time point.

Figure 3: (left) Models simulated incrementally shifting the WBA from the medial to lateral compartment; (right) Example of contact pressures on articulating tibial cartilage and meniscal surfaces. High pressures moved from medial to lateral compartment as WBA shifted laterally (all figures use same color threshold [0-4 MPa]; higher pressures in red/grey).

Figure 4: Averages and standard errors of normalized pressures on the (blue) medial and (red) lateral (top) cartilage and (bottom) meniscal surfaces. Proposed 'safe' zone shaded in grey. M-L = medial-lateral.

Figure A.5: Models were first created with the knee in its (left) native alignment (WBA force F shown in black). (right) Simulated HTO models were then created by shifting the WBA from the native alignment (black, F) towards the lateral compartment (red, F'). Shifting the WBA creates an adduction-abduction moment.

Figure B.6: Averages and standard errors of normalized von Mises stress 
573 and contact pressure on the articulating (solid lines) cartilage and (dashed 574 lines) meniscal surfaces of the (blue) medial and (red) lateral compartments. 575 Proposed 'safe' zone shaded in grey. M-L = medial-lateral.

576 Figure B.7: Relative (top) stresses and (bottom) pressures on the (blue) 577 medial and (red) lateral tibial cartilage and menisci. $\mathrm{M}-\mathrm{L}=$ medial-lateral. 